
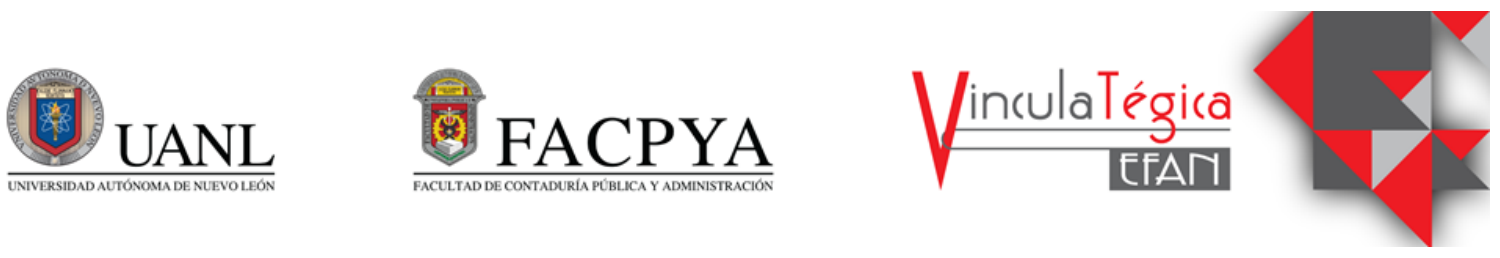

\title{
Retos del programa educativo ingeniero industrial administrador para el cumplimiento del mercado laboral ante la nueva era digital
}

\author{
Carolina Solís Peña ${ }^{1}$, Juan Manuel Hernández Ramos² y Iván Guillermo Palomo González ${ }^{3}$ \\ ${ }^{1}$ Universidad Autónoma de Nuevo León, carolina.solispa@uanl.edu.mx, Cd Universitaria, 83294000. \\ ${ }^{2}$ Universidad Autónoma de Nuevo León, juan.hernandezr@uanl.edu.mx, Cd Universitaria, 83294000. \\ ${ }^{3}$ Universidad Autónoma de Nuevo León, ivan.gonzalezpl@uanl.edu.mx, Cd Universitaria, 83294000. \\ Información del artículo revisado por pares \\ Fecha de aceptación: junio-2021 \\ Fecha de publicación en línea: diciembre-2021 \\ DOI: https://doi.org/10.29105/vtga7.1-81
}

\section{Resumen}

El mundo está cambiando constantemente lo cual trae como resultado formar profesionistas que puedan afrontar estos cambios. Muchas actividades dejaron de ser manuales para ser automatizadas y los procesos han dejado de requerir profesionistas para realizar estas actividades. La Era digital trae consigo el análisis de los datos con el objetivo de no cometer los mismos errores. El Ingeniero Industrial se dedica a la mejora de los procesos, generalmente se apoya de lápiz y papel para recabar información y hacer sus mejores, sin embargo, hoy en día es muy común que se apoye de las tecnologías de información para realizar el análisis de los datos (BIG DATA) recabados por las TIC y así mejorar los procesos. Debido a lo antes mencionado es muy importante que se generen egresados con las competencias que requiere el mercado para poder generar mejoras eficientes $y$ que hagan competitivas a las organizaciones. La presente investigación tiene como objetivo identificar primeramente de manera teórica cuales son los retos a los que el programa se enfrenta, para posteriormente desarrollar un instrumento de medición confiable para su replicación y además analizar los datos arrojados de la aplicación de una prueba piloto.

Palabras clave: era digital, Industria 4.0, Competencias, Mercado Laboral.

\begin{abstract}
The world is constantly changing which results in training professionals who can cope with these changes. Many activities stopped being manual to be automated and the processes have stopped requiring professionals to carry out these activities. The digital age brings with it the analysis of data with the aim of not making the same mistakes. The Industrial Engineers are dedicated to the improvement of processes, generally they rely on pencil and paper to gather information and make the improvements, however, nowadays it is very common that they rely on information technologies to perform the analysis of the data (BIG DATA) collected by ICT and thus improve processes. Due to the aforementioned, it is very important that graduates are generated with the skills required by the market in order to generate efficient improvements and make organizations competitive. The objective of this research is to firstly identify in a theoretical way what are the challenges that the program faces, to later develop a reliable measurement instrument for its replication and also to analyze the data obtained from the application of a pilot test.
\end{abstract}

Keywords: digital age, 4.0 industry, competencies, labor market.

JEL:I. 


\section{INTRODUCCIÓN}

Las organizaciones están cambiando constantemente con el objetivo de ofrecer siempre productos que cumplan con las necesidades del consumidor final. Para lograr lo mencionado, estas necesitan que las instituciones que se dedican a la formación del recurso humano ofrezcan programas no solo de calidad si no que estén siempre actualizados para las necesidades de la sociedad. En la presente investigación se pretende analizar las necesidades que tiene la sociedad con respecto al programa ingeniero industrial administrador, el cual al ser un agente de cambio debe de estar actualizado para cumplir con los requerimientos del mercado laboral.

A un estudiante de ingeniería industrial, se les enseña principalmente a analizar y diseñar sistemas productivos y los procedimientos de control para la operación eficiente de tales sistemas. El ingeniero industrial aporta un sentido de realidad comercial a los demás ingenieros. Establece un puente de comunicación a través del gran vació entre los gerentes y los ingenieros de desarrollo; es un traductor de las necesidades del cliente hacia la planta productiva; el ingeniero industrial analiza previamente las cosas de manera crítica, procura dar un paso atrás, a fin de observar mejor, en forma global, el ingeniero industrial es un amortiguador de personas, no solo trabaja las brillantes maquinas, sino también a sus operadores, estableciendo estándares de trabajo que sirvan como base de comparación, sugerirán formas de evaluar el trabajo y los planes viables de incentivos que utilizar; el ingeniero industrial es un protector de personas, se preocupa por la seguridad de sus trabajadores; es un planificador de plantas, ¿Cuánto?, ¿Cómo?, ¿Dónde?, busca mejoras y optimiza los procesos preguntándose ¿Es la mejor manera?, es un líder (Hicks, 2003).

Concluyendo lo ya mencionado se puede decir que la ingeniería industrial se ocupa del diseño, mejoramiento e instalación de sistemas integrados de personas, materiales y equipos; basándose en conocimientos y habilidades especializados en ciencias matemáticas, físicas y sociales, junto con los principios y métodos del análisis y diseño de ingeniería con el fin de especificar, pronosticar y evaluar los resultados que han de obtenerse de tales sistemas (Hicks, 2003).

\section{MARCO TEÓRICO}

En México la Ingeniería Industrial juega un papel muy importante en el soporte de la industria nacional, contribuyendo a su modernización y mejorando su nivel competitivo, tanto en el mercado interno como en los mercados internacionales (Ingeniería Industrial en cifras para México, 2020).

La carrera se registra oficialmente como tal en 1960 y, a partir de esa fecha, empieza a tener presencia en los diferentes estados de la República, conforme se creaban los Institutos Tecnológicos Regionales. Este tipo de sistema diseño los cursos para dar apoyo y soporte técnico al desarrollo regional.

Actualmente y tomando como referencia el cuestionario 911 de la Secretaría de Educación Pública, actualmente en el país la matrícula de los estudiantes de ingeniería es de 1,015,939 jóvenes para el periodo 20142015. En este reporte realizado por la SEP, se identificaron 33 áreas de ingeniería, siendo la ingeniería industrial la que encabeza la lista con 202,237 alumnos matriculados. El número total de programas que están relacionados a la ingeniería industrial que se imparten en México es alrededor del orden de 1248, ofertados en 1032 instituciones y estos se dividen en tres modalidades como puede ser observado en la tabla 1 (Cuestionario 911 de la SEP, 2016).

El ingeniero industrial se concibió como un profesionista cuya función básica era servir a la industria en provincia y así, arraigar a sus egresados a su lugar de origen (Roldan, Hanel, \& Echeverría, 2020). 
Tabla 1 Relación no. De programas / ofertados en instituciones /Matricula

\begin{tabular}{llll}
\hline Modalidad & No. De programas & Ofertados en instituciones & Matrícula \\
\hline Escolarizado & 1000 & 811 & 181,116 \\
No Escolarizado & 140 & 133 & 16,399 \\
Mixto & 108 & 88 & 4722 \\
\hline
\end{tabular}

Fuente: (Cuestionario 911 de la SEP, 2016).

De acuerdo a estudios realizados en Colombia se observa que el ingeniero industrial administrador se desarrolla en áreas como finanzas $(23.7 \%)$, comercio $(19.40 \%)$, manufactura $(15.80 \%)$, transporte y almacenamiento $(16.60 \%)$ y actividades profesionales, científicas y técnicas $(24.50 \%)$. Un ingeniero industrial debe de contar con los siguientes conocimientos y aptitudes: Cálculo (37.90\%), Diseño (17.40\%), Programación (18.60\%), Álgebra (17.80\%), Física (15.80 $\%)$, Calidad (58.50\%), Procesos (68\%), Logística y Producción (58.50\%) y otros (5.10 $\%)$. Para este último porcentaje se obtuvieron respuestas como, computación, programación $\mathrm{y}$ funcionalidad en maquinaria industrial, finanzas y proyectos, auditorías, estadística, investigación de operaciones, manejo de herramientas informáticas y herramientas como ERP o CRM, automatización de procesos financieros, de análisis de datos, ecomerce, $\mathrm{I}+\mathrm{D}$, así como administración del personal. En esta misma investigación se preguntó por la cantidad de prácticas en la empresa y de acuerdo a los resultados se indicó que al menos un $65 \%$ contaba con practicantes que estudian ingeniería industrial (Espitia, 2020).

Como se puede observar, la ingeniería industrial puede encontrarse en los diferentes procesos de producción de las empresas, con el diseño, el análisis, la instalación, la operación, la administración, el control, la calidad y la mejora continua de sistemas productivos y de servicios, integrados por personas, materiales, equipo, información y recursos financieros, con una visión de respeto al individuo, a la sociedad y al medio ambiente.

Relacionando lo antes mencionada el mundo industrial se renueva constantemente, debido a que las empresas de la era digital buscan incrementar su competitividad, pero sin disminuir sus ganancias. Para ello, se hace necesario optimizar procesos que permitan incrementar la productividad, lo cual inevitablemente requieren del trabajo de un ingeniero industrial. Estos profesionistas poseen una ventaja respecto a otro tipo de ingenieros: su capacidad de adaptación y formación para la toma de decisiones efectivas para el análisis de datos.

De acuerdo a la tendencia en el 2030 los y las ingenieros/as industriales serán profesionistas inmersos en organizaciones productivas, capaces de aplicar la innovación tecnológica con una fuerte formación holística y con gran capacidad para integrar conocimientos y resolver problemas complejos; una gran parte de su actividad seguirá siendo la optimización de recursos tecnológicos, humanos y materiales. Serán agentes de cambio en organizaciones con estructura horizontal, explotando las siguientes características: proactivos, innovadores, flexibles, aplicando las tecnologías de información y comunicación, generando un nuevo conocimiento, visión global, consciencia sustentable e integrando paradigmas inéditos (Roldan, Hanel , \& Echeverría, 2020).

Por lo cual en la actualidad la ingeniería industrial está relacionada directamente con la industria 4.0, término que hace referencia a Fabrica inteligente. En otras palabras, la aplicación del internet de las cosas a los procesos productivos y de servicios. Esto trae consigo un proceso de transformación digital, una nueva revolución industrial producida por el avance de las tecnologías de la información particularmente, de la informática y el software. En la figura 1 se observa una propuesta de Modelo de Negocios de Fabricación 4.0. Esta propuesta hace énfasis a los facilitadores tecnológicos presentes en los procesos de la organización. Se puede destacar el análisis de datos (Big Data), Plataformas Sociales, Impresión 3D, Robótica Colaborativa, así como Realidad Aumentada, como áreas en las que el ingeniero 
industrial administrador puede desarrollarse y las instituciones dedicadas a formar profesionistas por su parte deben de preocuparse por cumplir con esta demanda de la sociedad (del Van, 2016).

Figura 1 Modelo de Negocios de Fabricación 4.0

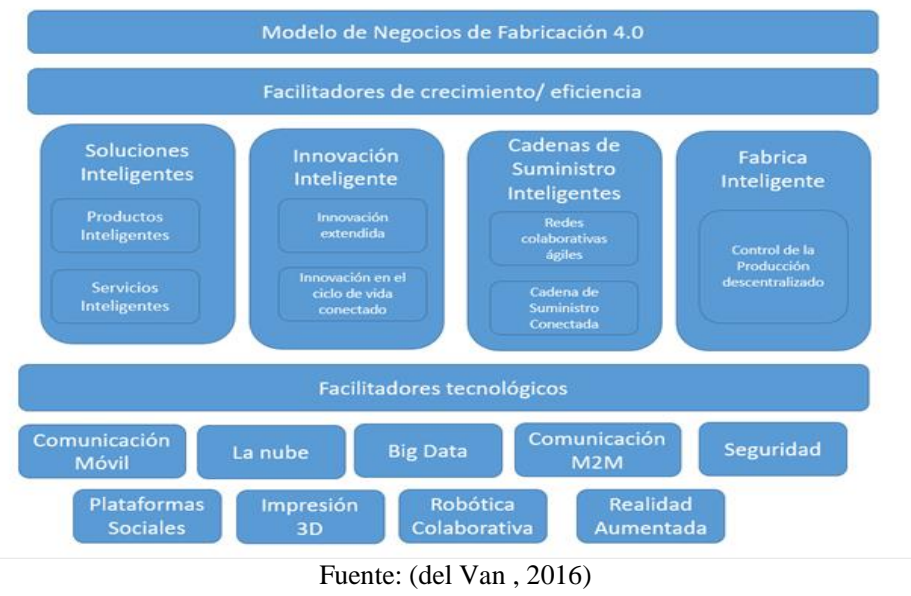

Con las investigaciones anteriores se puede concluir que las necesidades actuales del mundo, pasaron de requerir sistemas educacionales tradicionales a ser sistemas que involucren la industria 4.0 en sus procesos educativos.

La Educación 4.0 es un aprendizaje que se caracteriza por su flexibilidad y por la incorporación de manera integral el uso de las tecnologías de la información y las comunicaciones, la inteligencia artificial, analítica de datos, la gamificación en los procesos de enseñanza - aprendizaje, los métodos de autoformación, las habilidades blandas, entre otros. Además, la educación 4.0 se distingue por desarrollar la portabilidad de los contenidos educativos y las habilidades para ser aplicadas en diferentes entornos digitales, inmersivos, virtuales y ciber- físicos vinculados con el sector productivo y de servicios (Pascuas, Yois, Vargas, \& Muñoz, 2017) (Bodrow, 2017).
La Educación 4.0 también se identifica como la educación del futuro inmediato debido a la integración de la inteligencia artificial, Big Data, entre otras. Dentro de las principales características de la nueva educación en industria 4.0 se tiene las siguientes: 1) Programación científica como el nuevo lenguaje de comunicación entre los ingenieros y entre estos y las maquinas; 2) Desarrollo empresarial con enfoque en la innovación que facilitara la revolución de las tecnologías y 3) Aprendizaje analítico porque el conocimiento de lo intangible como las señales digitales serán de carácter obligatorio en todas las disciplinas.

De forma más concisa y establecida en la siguiente tabla 2 se muestran las competencias clásicas y las competencias industria 4.0, que deberá de desarrollar el ingeniero industrial administrador (Carvajal, 2017). 
Tabla 2 de competencias Clásicas vs Industria 4.0

\section{Competencias Clásicas}

Comprender y aplicar ciencias básicas y principios de ingeniería para resolver problemas

Modelar y solucionar problemas de ingeniería mediante el uso de las matemáticas

Analizar y verificar fenómenos físicos mediante la experimentación

Aplicar la estadística y la probabilidad para analizar e interpretar los resultados de procesos experimentales de ingeniería.

Diseñar algoritmos y programas de computación para solucionar problemas de ingeniería.

Concebir y Diseñar componentes, equipos y sistemas con base en los principios de ingeniería.

Examinar, formular y evaluar sistemas convergentes de Ingeniería.

Diseñar, implementar y operar sistemas convergentes de Ingeniería.

Aplicar Ingeniería en áreas multidisciplinarias, interdisciplinarias y transdisciplinarias.

Gestionar, formular y ejecutar proyectos de investigación en Ingeniería.

Hablar y escribir de acuerdo con las normas gramaticales correspondientes

Aplicar principios de ética y bioética en su desempeño profesional

Formación profesional en las Instituciones de Educación Superior IES

Fuente: Carvajal, 2017.

Como se puede observar la Educación 4.0 es un proceso de transformación en donde las TIC, inteligencia artificial, analítica de datos, educación a distancia, ludificación, portabilidad, entre otras aplicables a todos los modelos y niveles educativos. Este aprendizaje debe de ser flexible, adaptativo, retroalimentado, individualizado y colaborativo. El aprendizaje debe de poder impartirse de manera presencial o virtual de acuerdo a la necesidad de la unidad de aprendizaje a impartir. Esto con el objetivo de cumplir con las necesidades del cliente final en este caso la industria, los sistemas de enseñanza, las sociedades, la mejora de la calidad de vida y la sustentabilidad.

Para el éxito de la Industria 4,0 el entrenamiento y la cualificación de los tecnólogos y profesionales universitarios serán adaptados a los nuevos requerimientos de producción digital. Entre las habilidades
Competencias Industria 4.0

Aprender a comprender y aplicar software especialista en la modalidad e-learning

Usar Matlab - Simulink o programas afines para solucionar problemas de ingeniería.

Analizar y experimentar a través de laboratorios remotos, virtuales e interactivos.

Operar MatLab - Simulink, Mathematics o softwares afines como herramienta e- learning

Concebir, diseñar, implementar y operar lógica de programación y computación en estaciones digitales de trabajo y su conexión a redes de comunicaciones

Concebir, diseñar, implementar y operar herramientas

CAD/CAM/CAE/FEA para modelado y simulación de elementos, dispositivos y sistemas de ingeniería.

Integración sinérgica de multisistemas por medio de ICT asistidas por computador.

Concebir, diseñar, implementar y operar sistemas complejos de ingeniería por computadores digitales.

Concebir, diseñar, implementar y operar sistemas

complejos usando ICT integrados por computadores digitales

Gestionar y mantener en equipo interdisciplinario sistemas multidisciplinares de ingeniería en laboratorios virtuales, remotos e interactivos.

Emplear e- learning para aprender, conversar y escribir con normas gramaticales correspondientes

Juzgar, valorar y evaluar principios de ética y bioética en ambiente virtual.

Formación profesional en las Instituciones de Educación Superior IES con pasantías en las Fábricas Digitales

requeridas se distinguen: Aplicación de dispositivos móviles como Tablets y Smarthphones en la producción; Planeamiento y organización de redes de trabajos de componentes y módulos de sistemas inteligentes, Uso del ERP, integración virtual de estaciones de producción a través de la internet. Y entre las capacidades que se deben de tener es la adaptación a los cambios de trabajo en la Fabrica digital debido a la evolución // Innovación de las tecnologías, análisis y evaluación de datos de los procesos; Integración digital de los componentes y de módulos dentro de un sistema de comunicación complejo entre otros.

Además de lo ya mencionado los programas serán desarrollados en cooperación con el sector empresarial o las universidades y los empresarios serán socias para el desarrollo de los programas de ingeniería, los planes curriculares serán comprimidos y 
complementados con pasantías empresariales, los contenidos curriculares se desarrollarán a profundidad complementados con conocimientos sociales y tecnológicos.

Esta investigación se tiene como objetivo probar la confiabilidad del instrumento de medición, y posteriormente analizar mediante el análisis de graficas de pastel las respuestas obtenidas en la aplicación del documento.

El modelo usado en la presente investigación es el que se muestra en la figura 2.

Figura 2 Modelo Propuesto (Regresión Lineal Múltiple)

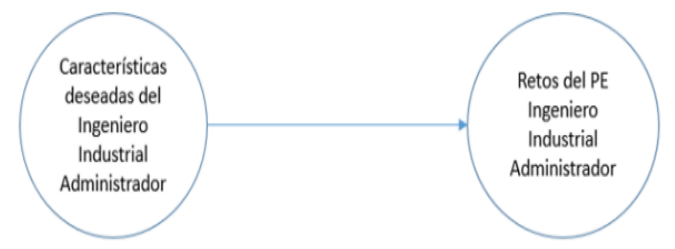

Fuente: Elaboración Propia del Autor.

\section{MÉTODO}

Esta investigación es de carácter cuantitativo, no experimental, transversal. Para la recolección de datos se apoyó a través de un instrumento de medición, en formato tipo encuesta (Sampieri , 2018). Se tomó la decisión de aplicar el instrumento de medición a los egresados de los últimos 5 año de ingeniería industrial, lo cual da como resultado 1802 (considerando que la investigación se realizado durante el primer semestre del año 2020), ya que actualmente son ellos los que se encuentran laborando en las instituciones hoy en día, y apoyan a la vinculación de la institución de educación superior con el campo laboral.

Tabla 3 de sustentantes por semestre

\begin{tabular}{|c|c|}
\hline Año & \multirow[t]{2}{*}{ Número de Sustentantes EGEL } \\
\hline Semestre & \\
\hline 2015 (1) & 224 \\
\hline $2015(2)$ & 106 \\
\hline $2016(1)$ & 215 \\
\hline $2016(2)$ & 173 \\
\hline $2017(1)$ & 241 \\
\hline $2017(2)$ & 104 \\
\hline 2018 (1) & 221 \\
\hline $2018(2)$ & 128 \\
\hline 2019 (1) & 251 \\
\hline $2019(2)$ & 139 \\
\hline
\end{tabular}

\subsection{Calculo de la muestra}

La muestra es un subgrupo de la población, en otras palabras, es un subconjunto de elementos que componen un todo. Para determinar el valor de la muestra el nivel de confianza que se usa es del 95\%, lo cual nos indica que tenemos un error del 5\%. Al aplicar la formula se obtuvo que la muestra a usar la presente investigación seria de: 317 egresados del plan de estudios de ingeniero industrial.

\subsection{Método de análisis estadístico}

En la presente investigación se usará la regresión lineal simple, ya que solo se tiene 
una variable dependiente y una independiente (Hair, Black, Babin, \& Anderson, 2014). Un modelo de regresión múltiple que puede describir esta relación se encuentra en la ecuación 1 :

\section{Ecuación 1 Regresión lineal múltiple}

$Y_{1}=\beta_{0}+\beta X_{1}+\beta X_{2}+\beta X_{3}+\ldots \ldots+\beta X_{n}+\epsilon$

Fuente: (Hair, Black, Babin, \& Anderson, 2014)

En donde:

$\mathrm{Y}=$ Valor estimado para la variable dependiente

$\beta=$ Coeficientes parciales (o netos) de regresión.

$\mathrm{X}_{1}, \mathrm{X}_{2} \ldots \mathrm{X}_{\mathrm{n}}=\mathrm{Las}$ variables de la ecuación

$\epsilon=$ Error que cometemos en la predicción de

los parámetros.

El coeficiente de las Betas es un estadístico que nos indica la correlación que hay entre el comportamiento de una variable y otra. En esta investigación se tomó la decisión de multiplicar los constructos de cada una de las " $X$ " con el objetivo de explicar el modelo en porcentajes.

Sin embargo, como se mencionó en el objetivo de la investigación solo se validará la confiabilidad del instrumento de medición y se analizaran los resultados mediante grafica de pastel.

\section{RESULTADOS}

Para realizar el análisis de la confiabilidad del instrumento de medición se utilizó el alpha de cronbach. La confiabilidad de un instrumento hace referencia "al grado en que su aplicación repetida al mismo individuo u objeto produce resultados iguales" Para evaluar la confiabilidad del instrumento se utilizó como ya se mencionó el alpha de cronbach el cual determina la correlación de cada reactivo de la variable X con el grupo de la variable Y. Para interpretar los resultados obtenidos del alpha de cronbach se establece lo siguiente: Una alpha con valor de .70 es lo mínimo aceptable, .80 es bueno y de .90 es excelente (Celina \& Campo, 2005).

Para comprobar la confiabilidad del instrumento de medición, se realizó una prueba piloto en donde se aplicó el instrumento de medición a 233 estudiantes, de lo cual podemos destacar lo mostrado en la tabla \#\#, en donde se demuestra que las dos variables se encuentra el parámetro excelente, con esto se puede concluir que el instrumento de medición es confiable.

Tabla 4 Resultados de Alpha de Cronbach

\begin{tabular}{ccc}
\hline Variable & Resultado & Se eliminó algún Ítem \\
\hline Retos del Plan de Estudios de IIA & .900 & Ninguno \\
Características deseadas del IIA & .910 & Ninguno
\end{tabular}

Fuente: Elaboración Propia del Autor con apoyo del SPSS.

A pesar de la gran cantidad de encuestas aplicadas, se consideró realizar un análisis factorial únicamente exploratorio con el objetivo de determinar con mayor precisión las dimensiones subyacentes, constructos o variables latentes que pudieran afectar la ecuación del modelo propuesto. Se consideró que fuera exploratorio por el hecho de que, si un ítem cargaba en un constructo que no debiera de ser, este fuera eliminado. La situación antes mencionada sucedió con el ítem CDI18, el cual cargaba en la variable contraria como puede ser observado en la tabla 5. Es necesario mencionar que este ítem fue eliminado y trae como resultado la aplicación nuevamente del instrumento de medición. En la tabla 6 se muestra en donde cargan de manera correcta los ítems propuestos ya eliminando el ítem CDI18. 
Tabla 5 Análisis Factorial Exploratorio con ítem CDI18 cargando en la variable dependiente.

\begin{tabular}{lll} 
& Componente \\
RPEI & 1 & 2 \\
RPE2 & .316 & .706 \\
RPE3 & .209 & .740 \\
RPE4 & .276 & .772 \\
RPE5 & .209 & .764 \\
RPE6 & .234 & .776 \\
RPE7 & .251 & .716 \\
RPE8 & .354 & .516 \\
RPE9 & .441 & .528 \\
CDI10 & .539 & .547 \\
CDI1 & .633 & .384 \\
CDI12 & .749 & .255 \\
CDI13 & .757 & .311 \\
CDI14 & .809 & .208 \\
CDI15 & .672 & .210 \\
CDI16 & .837 & .210 \\
CDI17 & .696 & .410 \\
CDI18 & .590 & .393 \\
\hline
\end{tabular}

Fuente: Elaboración Propia con apoyo del SPSS.

Tabla 6 Análisis Factorial Exploratorio sin ítem CDI18

\begin{tabular}{lll} 
& \multicolumn{2}{l}{ Componente } \\
RPEI & 1 & 2 \\
RPE2 & .321 & .710 \\
RPE3 & .214 & .746 \\
RPE4 & .281 & .770 \\
RPE5 & .214 & .769 \\
.RPE6 & .239 & .777 \\
RPE7 & .256 & .714 \\
RPE8 & .359 & .507 \\
RPE9 & .443 & .529 \\
CDI10 & .541 & .544 \\
CDI11 & .636 & .384 \\
CDI12 & .750 & .264 \\
CDI13 & .758 & .312 \\
CDI14 & .810 & .207 \\
CDI15 & .674 & .197 \\
CDI16 & .838 & .205 \\
CDI17 & .700 & .401 \\
& .594 & .378
\end{tabular}

Fuente: Elaboración Propia con apoyo del SPSS.

Es necesario mencionar que en ambas corridas se cumple con el test de esfericidad de Barlett, le cual consiste en una estimación de jicuadrada a partir de la transformación del determinante de la matriz de correlaciones. Si las variables no están intercorrelacionadas, entonces el test de esfericidad de Barlett debe de presentar un valor de significancia superior 
al límite de .05. Para esta investigación se presentó una significancia muy inferior al límite .05 , pues fue de .000 , lo cual nos indica que la matriz de datos es válida para continuar con el proceso de análisis factorial. Para el caso del índice de Kaiser-Meyer-Olkin el cual mide la adecuación de la muestra. Indica que tan apropiado es aplicar el Análisis factorial. Cuanto más cerca se tenga el valor a 1 el KMO interpreta que la relación entre las variables es alta. Si el KMO es mayor o igual a .9 el test indica que es muy bueno, mayor a .8 es notable y mayor a .7 es mediano. Para esta investigación el resultado se encontró en .943 , lo cual nos indica que es apropiado el uso del análisis factorial, estos resultados pueden ser apreciados en la tabla 7.

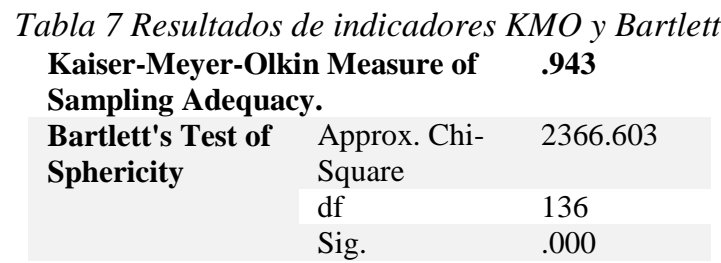

Fuente: Elaboración Propia del autor con apoyo del SPSS.

Con lo mencionado anteriormente se puede concluir que el instrumento de medición es confiable, por lo cual se puede seguir aplicando a la muestra restante.

Por otro lado, el análisis mediante grafico de sectores de los ítems más representativos del instrumento de medición nos dará una perspectiva general de la opinión del sujeto de estudio.

El ítem 1 hace referencia a la siguiente pregunta "Considera que el programa de ingeniero industrial contribuye a formar profesionistas que satisfagan las necesidades actuales de la sociedad", los resultados presentados se muestran en la figura 3 en donde un $47 \%$ indica que está totalmente de acuerdo y solo un $2 \%$ totalmente en desacuerdo. Lo cual nos indica que la gran mayoría está de acuerdo que el programa es pertinente con el contexto actual en el que se desarrolla.

Figura 3. Distribución de respuestas para el Item 1.

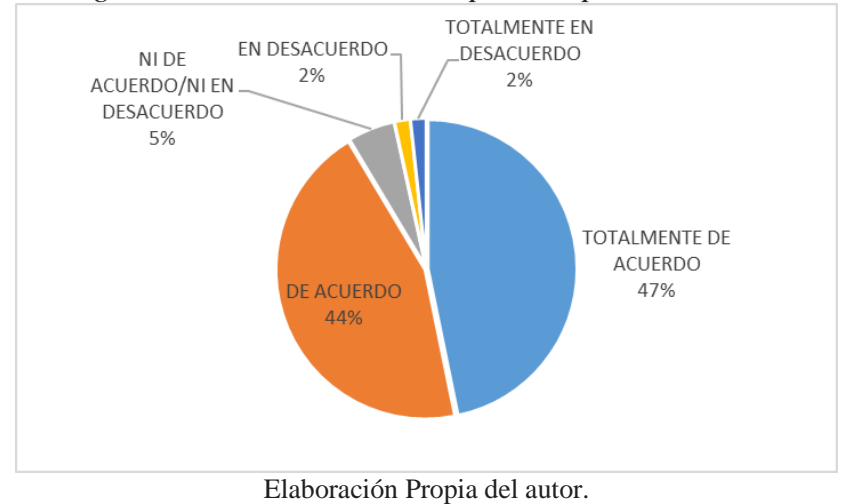

El Ítem 2 hace referencia a la siguiente pregunta "Considera que el programa de ingeniero industrial contiene características correspondientes al aprendizaje flexible", Al menos un $35 \%$ está totalmente de acuerdo, un $47 \%$ de acuerdo y solo un $2 \%$ está en desacuerdo, lo cual indica que el PE Ingeniero Industrial, ya que permite que el estudiante pueda aprender mediante audios, conferencias, textos, en cuanto a la opción de tiempo puede ser en tiempo real o diferido y en la opción de lugar puede ser desde casa o 
en las aulas. Además, se considera flexible datos pueden ser observados en la figura 4 . porque contiene una serie de materias optativas que le permiten al estudiante especializarse en el área de su interés. Estos

Figura 4. Distribución de respuestas para el Item 2.

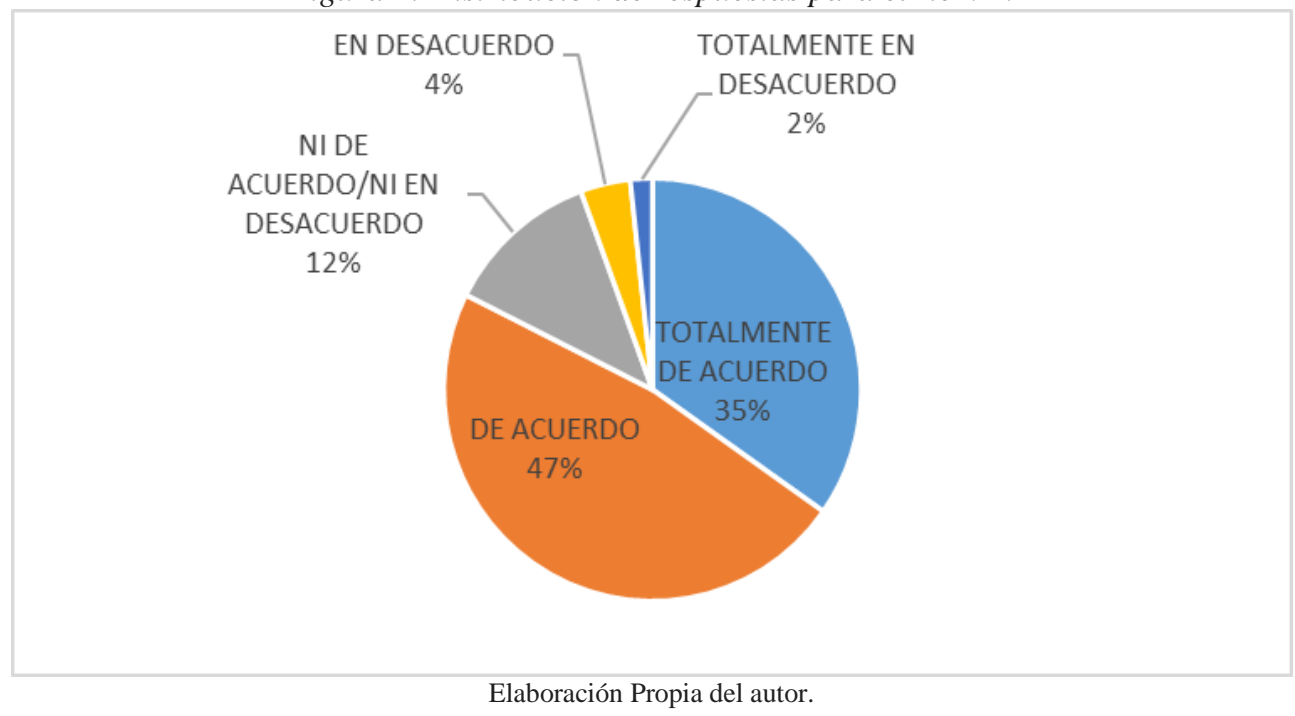

El Ítem 4 hace referencia al enunciado en donde se interroga si el PE fomenta el trabajo colaborativo, podemos destacar que los al menos un $84 \%$ de los encuestados indica que efectivamente el PE, fomenta el trabajo colaborativo, lo cual habla muy bien del programa ya que hoy en día es muy importante el trabajo entre equipos multidisciplinarios. Estos resultados pueden ser observados en la figura 5.

Figura 5. Distribución de respuestas para el Item 4.

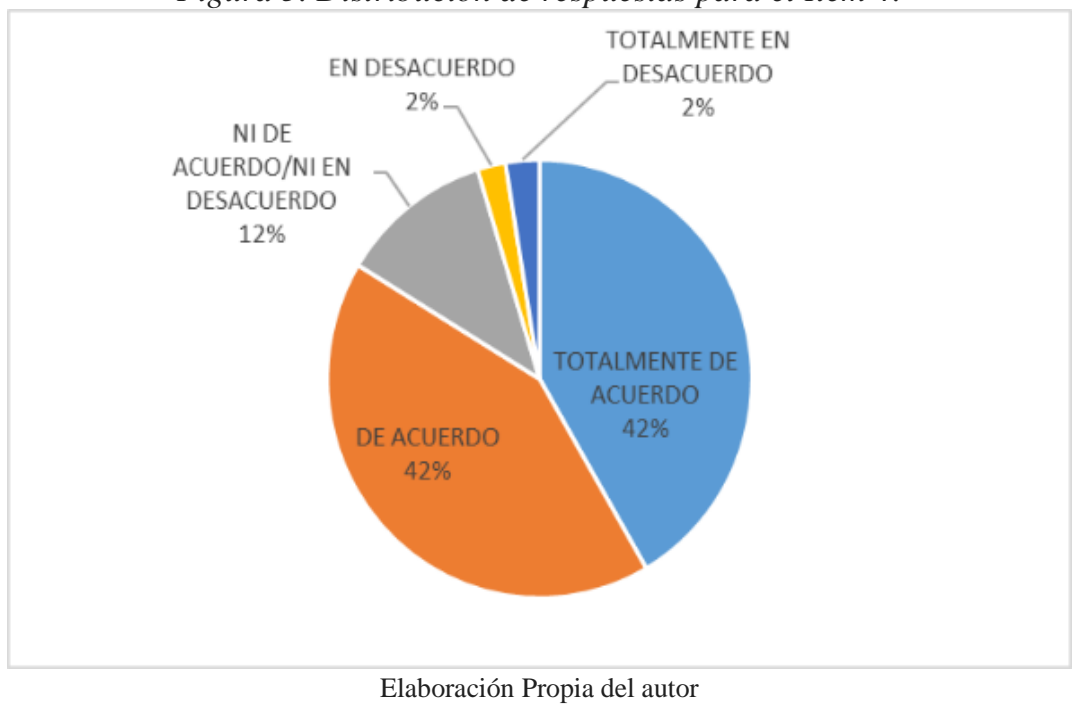

Para el Item 5 el cual hace referencia al desarrollo de talentos, al menos un $73 \%$ está de acuerdo, esto puede ser observado en la figura 6. 
Figura 6. Distribución de respuestas para el Item 5.

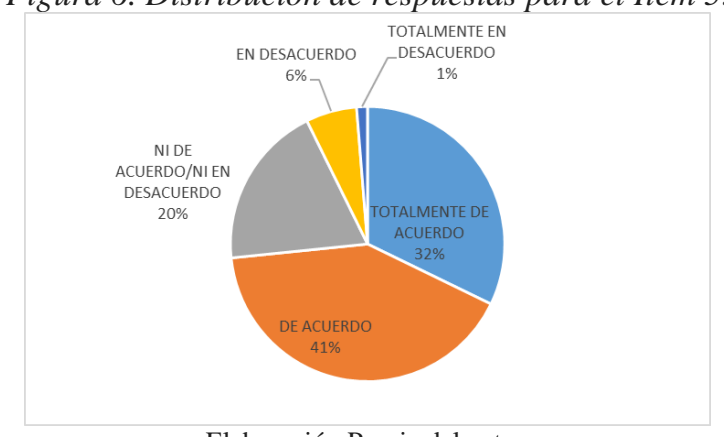

Elaboración Propia del autor.

El Item 6 hace referencia a impulsar la innovación, los encuestados refieren que al menos un $73 \%$ está de acuerdo, un $22 \%$ manifestó no estar ni de acuerdo/ni en desacuerdo, lo cual llama la atención. En este ítem es necesario revisar que consideran ellos como Innovación, el programa de estudios actualmente cuenta con una UA enfocada a la innovación únicamente tal vez los resultados se vieron influenciados por considerar que la innovación solo se desarrolla en esa UA. Los demás resultados pueden ser observados en la figura 7 .

Figura 7. Distribución de respuestas para el Item 6.

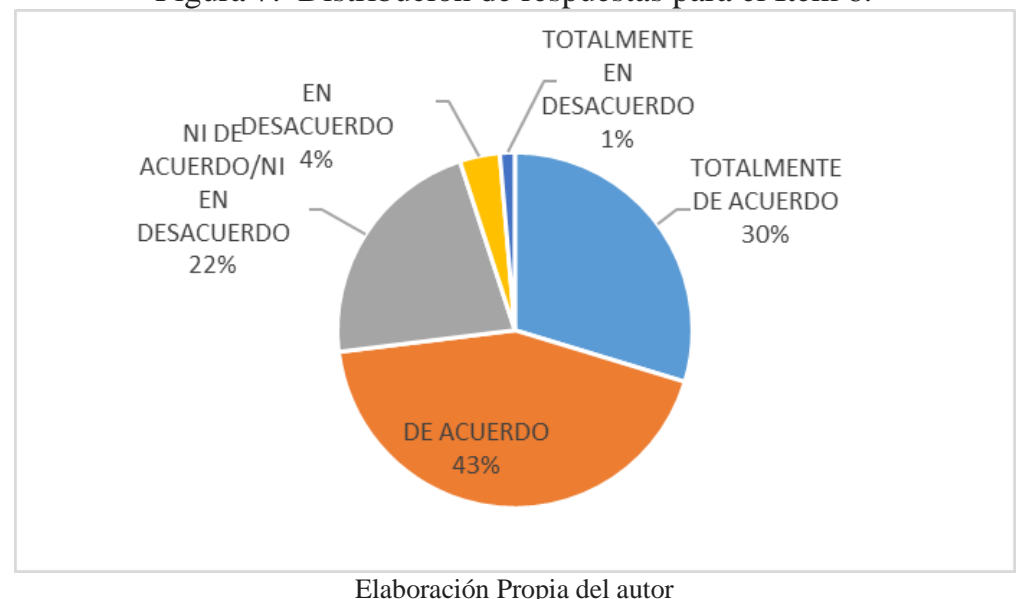

El Ítem 7 hace referencia a fomentar el emprendimiento, de acuerdo a los resultados mostrados en la figura 8 se puede destacar que al menos un $69 \%$ está de acuerdo en que el PE fomenta el emprendimiento. Y un 31 \% no está de acuerdo, ni de acuerdo o está orientado al lado de desacuerdo. Con estos resultados se recomienda revisar el Plan de estudios con el objetivo de fomentar el emprendimiento en los egresados. 
Figura 8. Distribución de respuestas para el Item 7.

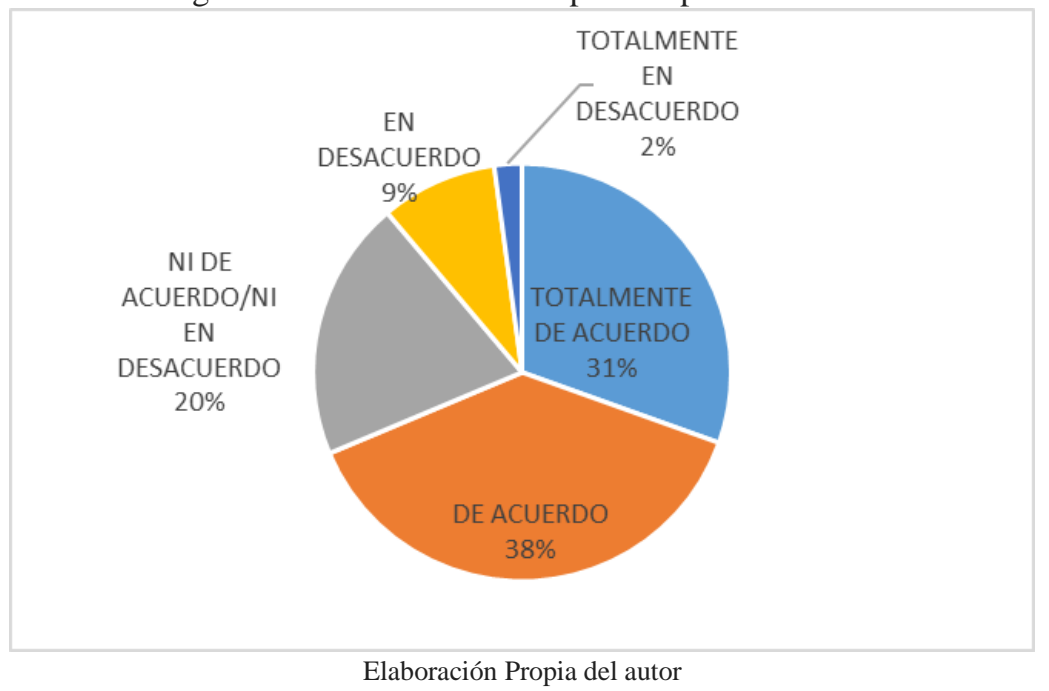

El Ítem 8 nos habla acerca de la colaboración entre la institución educativa con las empresas y centros de desarrollo tecnológico para el desarrollo de nuevos proyectos. En la figura 9 se puede observar que al menos un $66 \%$ está afirma que si existe esta colaboración y el $44 \%$ indica que no está de acuerdo o que no sabe si existe esta colaboración.

Figura 9. Distribución de respuestas para el Item 8.

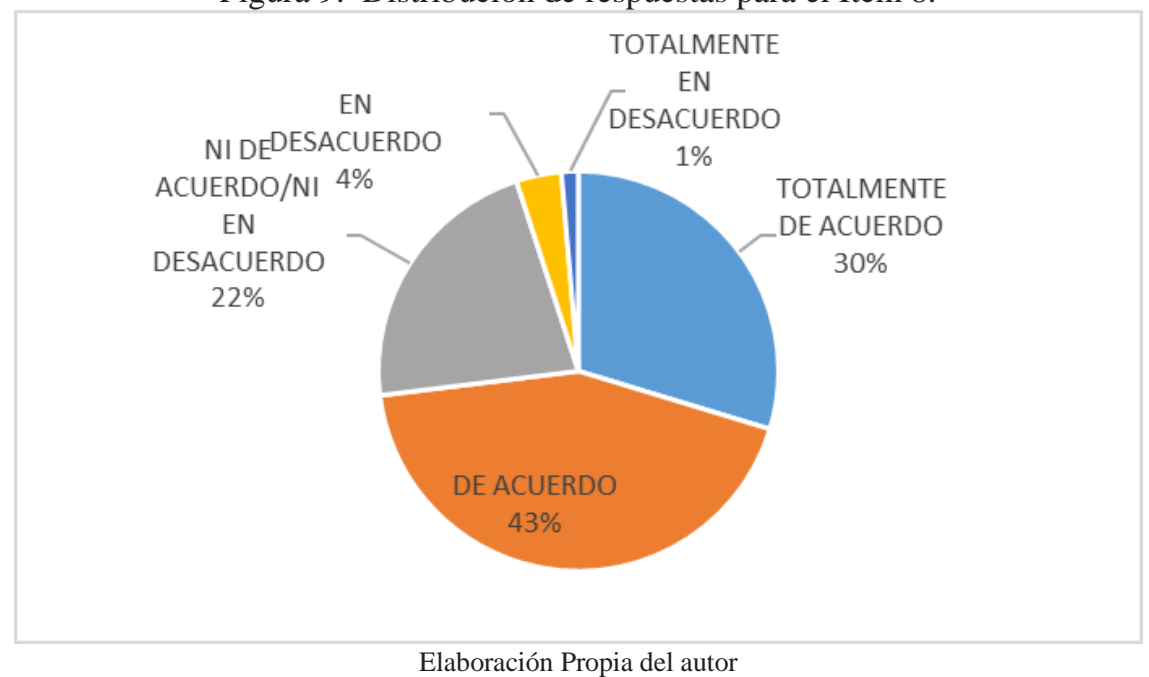

El Ítem 9 habla sobre el desarrollo del ingeniero industrial en la industria 4.0, al menos un $56 \%$ está de acuerdo según los datos presentados en la figura 10. Lo interesante en esta pregunta, es investigar que es para ellos industria $4.0 \mathrm{y}$ en donde visualizan a un IIA. 
Figura 10. Distribución de respuestas para el Item 9.

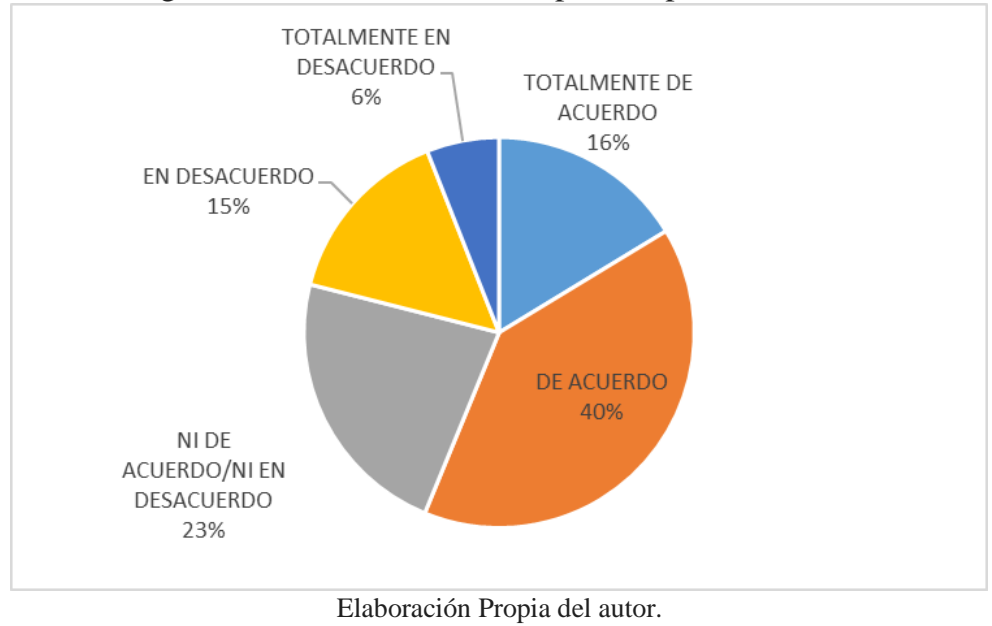

El Ítem 10 al igual que el pasado están

en la figura 11 en donde nuevamente un $56 \%$ relacionados con la industria 4.0, por lo cual está de acuerdo o totalmente de acuerdo. se puede observar un resultado similar, esto

Figura 11. Distribución de respuestas para el Item 10.

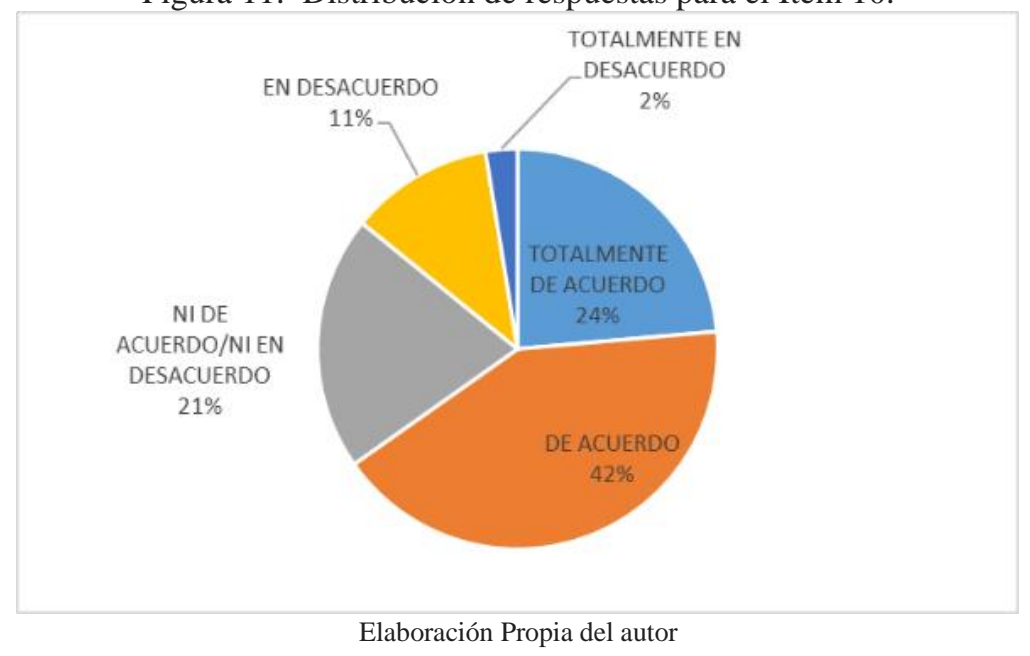

El Ítem 11 habla sobre el uso de softwares para el análisis de datos, estos incluyen la programación como elemento indispensable para su uso. Podemos observar que a diferencia de las preguntas anteriores por encima del $62 \%$ contesto que no estaban preparados para manejar estas herramientas.
Por lo cual se considera un área de oportunidad para el plan de estudios actual, esto mostrado en la figura 12. 
Figura 12. Distribución de respuestas para el Item 11.

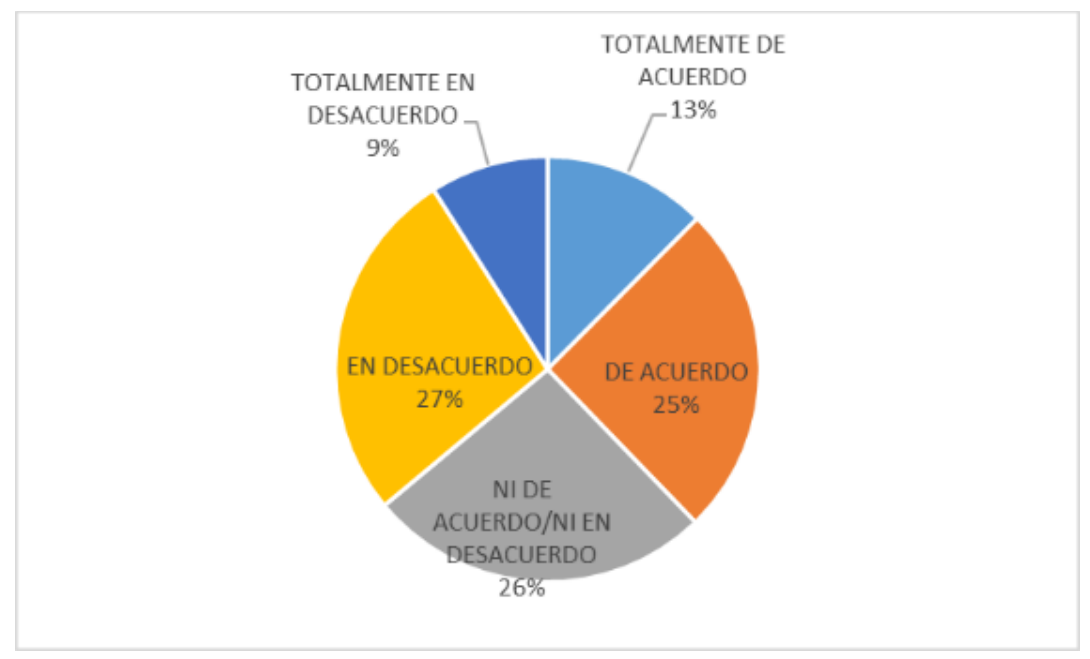

Elaboración Propia del autor

A diferencia del Ítem 11, en este (ítem 12) el cual hace referencia al análisis y experimentación en laboratorios virtuales y remotos, al menos un $60 \%$ de los encuestados manifestaron tener la competencia mencionada, mostrado en la figura 13.

Figura 13. Distribución de respuestas para el Item 12.

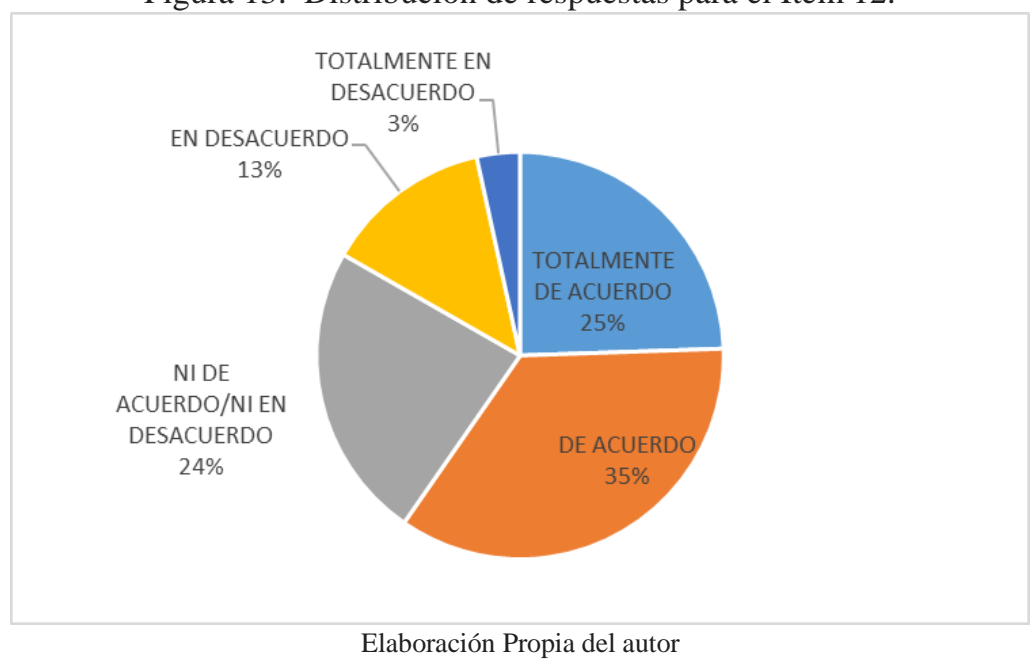

El Ítem 13 evalúa la capacidad de diseñar, implementar y manejo de equipo de cómputo en estaciones digitales. $\mathrm{Al}$ menos un $56 \%$ de los encuestados manifestaron tener esta competencia. (Figura 14) 
Figura 14. Distribución de respuestas para el Item 13.

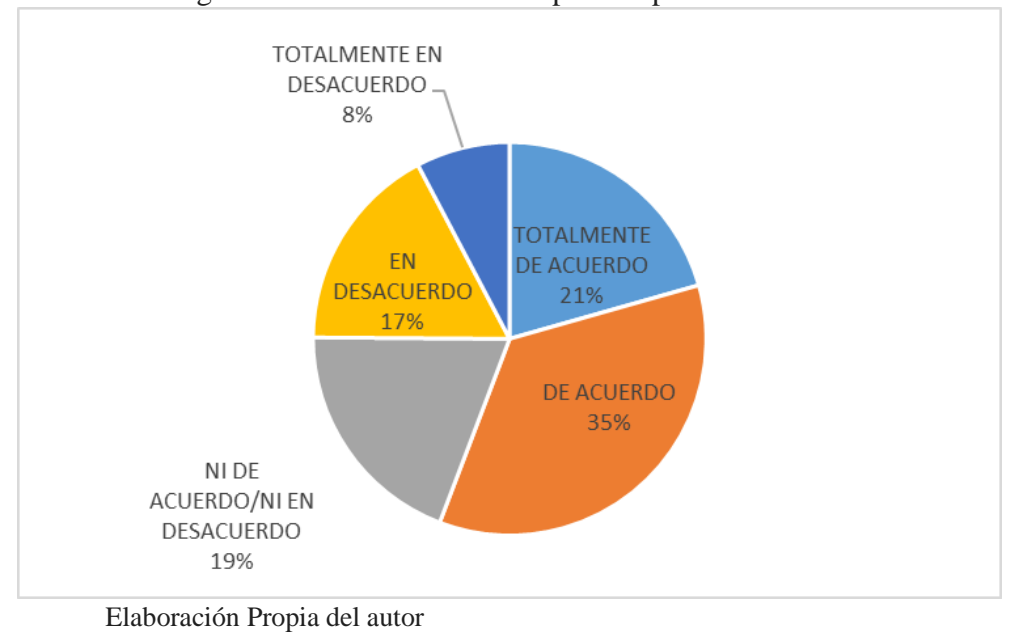

Elaboración Propia del autor

Con respecto al Ítem 14, en el cual se menos un $66 \%$ indico estar de acuerdo en reunir esta competencia. (Figura 15) habla sobre el uso de softwares de diseño, al

Figura 15. Distribución de respuestas para el Item 14.

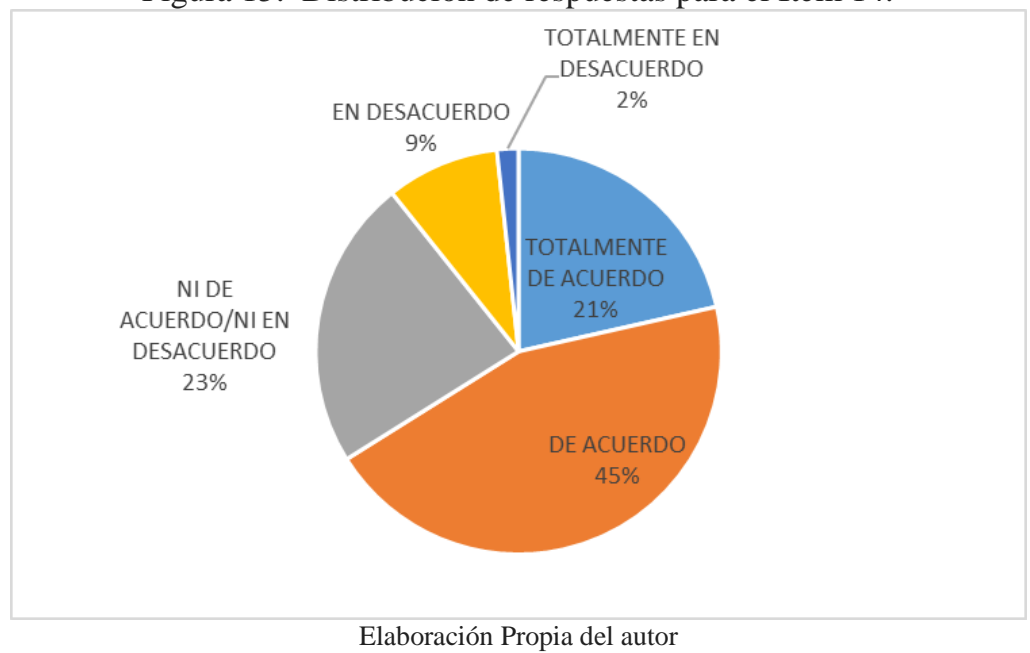

El ítem 15 habla sobre la capacidad del ingeniero industrial para concebir, diseñar, implementar y operar sistemas complejos de ingeniería. Los resultados muestran que al menos un $62 \%$ se considera capaz de poder desarrollar esta tarea y el $38 \%$ restante no sabe o no está de acuerdo en tener esta competencia (Figura 16). 
Figura 16. Distribución de respuestas para el Item 15.

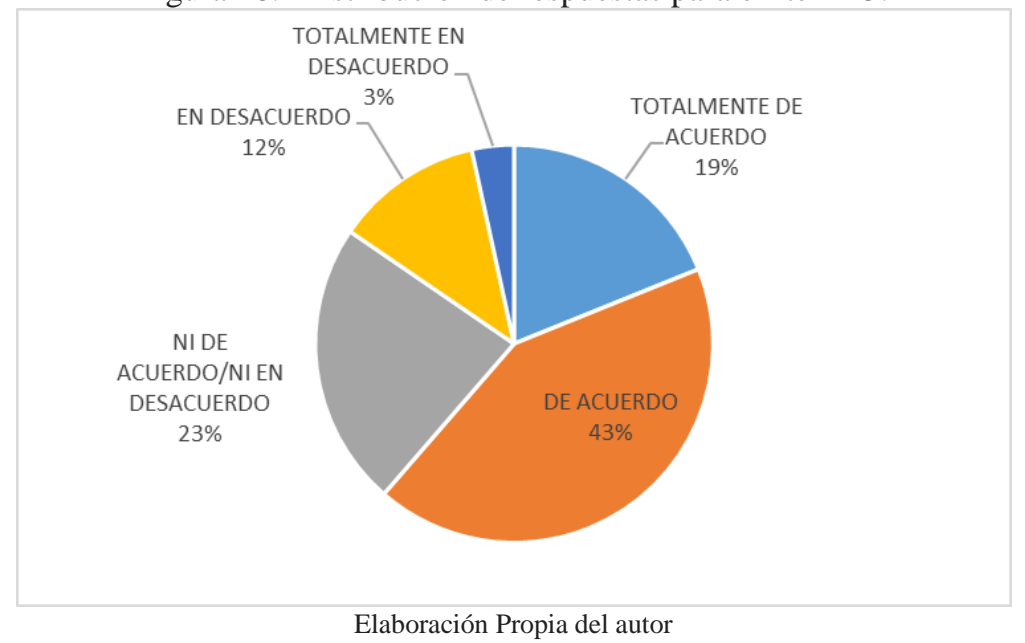

El Ítem 16 habla sobre poder gestionar desarrollar esta actividad. (Figura 17). equipos interdisciplinarios de manera virtual. Los resultados mostraron que al menos un $76 \%$ se encuentra capacitado para

Figura 17. Distribución de respuestas para el Item 16.

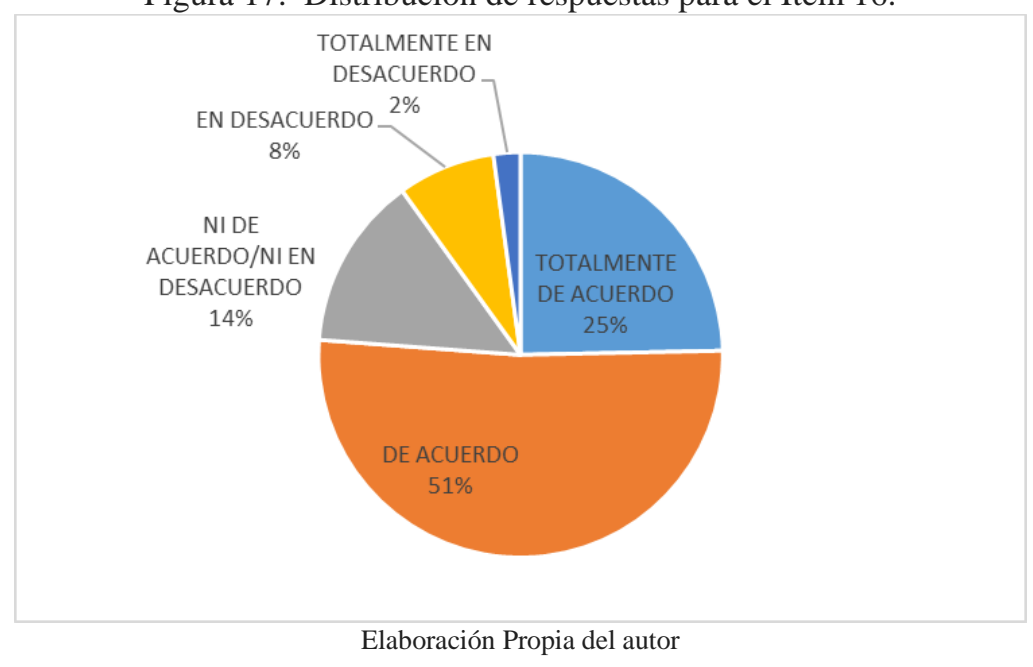

El Ítem 18 habla sobre la ética en el estos valores. (Figura 18) ejercicio de la profesión en este ítem al menos el $80 \%$ está de acuerdo que se tienen 
Figura 18. Distribución de respuestas para el Item 18.

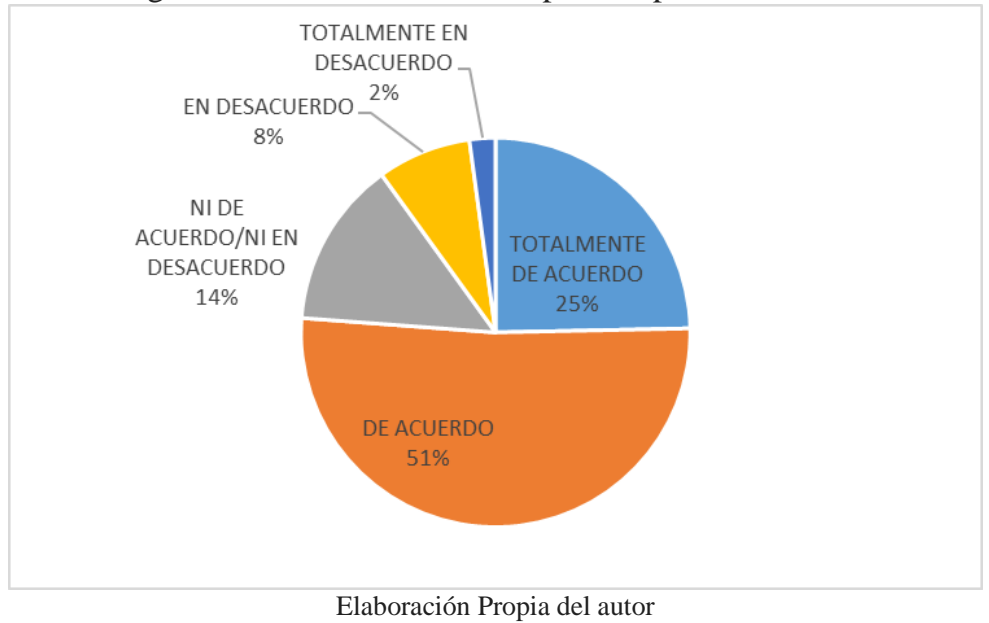

\section{CONCLUSIONES}

Debido a la naturaleza del estudio que se está realizando, se logró comprobar la confiabilidad del instrumento de medición, además se realizó un análisis factorial exploratorio para corroborar la correcta carga de los ítems en cada uno de los constructos. Por lo tanto, se puede concluir que el instrumento de medición puede ser aplicado al resto de la muestra con el objetivo de determinar el modelo que representaría el estudio realizado. Con respecto al análisis de las respuestas de cada uno de los ítems, se puede concluir que la mayoría contesta estar totalmente de acuerdo o de acuerdo, solo algunos ítems están en ni desacuerdo y desacuerdo, lo cual trae como resultado tomar esta información para la reestructuración del plan de estudios o el cambio en los temas de las unidades de aprendizaje optativas. También es necesario recalcar que el sujeto de estudio fueron aquellas que cursan el plan 2012 y el plan de estudios ya sufrió dos reestructuraciones, por lo que sería interesante aplicarlo posteriormente a las nuevas generaciones. 


\section{REFERENCIAS}

Bodrow, W. (2017). Impact of Industry 4.0 in service oriented firm. Advances in Manufacturing, 17.

Carvajal, J. (2017). La cuarta revolución industrial o industria 4.0 y su impacto en la educación en ingeniería en latinoamerica y el caribe. 15th h LACCEI International Multi-Conference for Engineering, Education, and Technology, 1-5.

Celina, H., \& Campo , A. (2005). Aproximación al uso del coeficiente alfa de Cronbach. Revista Colombiana de Psiquiatría, 572-580.

(2016). Cuestionario 911 de la SEP.

del Van , J. (2016). Industria 4.0: La transformación digital de la industria. España: coddiinforme.

Espitia, S. (2020). Analisis de las necesidades y requerimientos de entorno laboral en el 2020 a nivel local y regional de un ingeniero industrial. Colombia: Universidad Catolica de Colombia.

Hair, J., Black, W., Babin, B., \& Anderson, R. (2014). Multivariate Data Analysis. England: Pearson.

Hernandez Sampieri, R. (2014). Metodologia de la Investigacion. Mc Graw hill.

Hicks, P. (2003). Ingeniería Industrial y Administración. Una nueva perspectiva. México: CECSA. Ingenieria Industrial en cifras para México. (2020). Obtenido de Universia.

Pascuas, R., Yois, S., Vargas, E., \& Muñoz, J. (2017). Experiencias Motivacionales gamificadas: una revisión sistemática de literatura. Innovación Educativa, 63-80.

Roldan , J., Hanel , M., \& Echeverría, J. (2020). ingeniería Industrial en México 2030: Escenario de futuro. Estudio de Planeación Prospectiva.

Sampieri , R. (2018). Metodología de la investigación: las rutas cuantitativa, cualitativa y mixta . México: Mc Graw Hill. 